\title{
Challenges and solutions to sharing a cancer follow-up e-care plan between a cancer service and general practice
}

\author{
Jane Taggarta,j, Melvin Chin ${ }^{\mathrm{b}, \mathrm{c}}$, Winston Liauw ${ }^{\mathrm{d}, \mathrm{e}, \mathrm{f}}$, David Goldstein ${ }^{\mathrm{b}, \mathrm{c}, \mathrm{f}}$, \\ Alex Dolezal' , John Plahn' and Mark F Harrisa,f,g \\ a Centre for Primary Health Care and Equity, UNSW Sydney, Australia \\ b Nelune Comprehensive Cancer Centre, Prince of Wales Hospital, South Eastern Sydney Local Health District, NSW, Australia \\ c Prince of Wales Clinical School, Faculty of Medicine, UNSW Sydney, Australia \\ d Cancer Services, South Eastern Sydney Local Health District, NSW, Australia \\ e St George Hospital Cancer Care Centre, Sydney, NSW, Australia \\ f Translational Cancer Research Network, UNSW Sydney, Australia \\ g School of Public Health and Community Medicine, UNSW Sydney, Australia \\ ${ }^{\mathrm{h}}$ Central and Eastern Sydney Primary Health Network, Sydney, NSW, Australia \\ eHealth NSW, New South Wales Ministry of Health, Sydney, Australia \\ j Corresponding author: J.Taggart@unsw.edu.au
}

\section{Article history}

Publication date: June 2021

Citation: Taggart J, Chin M, Liauw W, Goldstein D, Dolezal A, Plahn J, Harris MF. Challenges and solutions to sharing a cancer follow-up e-care plan between a cancer service and general practice. Public Health Res Pract. 2021;31(2):e31122108. First published 30 April 2021. https://doi. org/10.17061/phrp31122108

\section{Abstract}

Objective: This paper describes the process of developing a shared cancer care approach in follow-up, and identifies the e-health options that support an interactive e-care plan shared between a public cancer service, general practitioners (GPs) and cancer survivors.

Type of program/service: The cancer service improvement initiative for shared care in follow-up targets colorectal cancer patients who have completed active treatment and who agree to shared care between specialists, GPs and other care team members. The intiative is supported by an agreed shared care pathway and an interactive e-care plan that is dynamic, can be shared and has functionalities that support collaboration.

Design and development: A consultative process with stakeholders (local and state health services, a Primary Health Network, GPs and a consumer) was undertaken. Responses from individual consultations (25 stakeholders) were collated and commonalities identified to inform a workshop with 13 stakeholders to obtain consensus on the care pathway and e-health solution. Implications for policy and practice were identified throughout the process.

Outcomes: The stakeholders agreed to a shared care pathway, which included assessment and consent, GP engagement, tailoring the care plan and communicating results and information as tasks are completed. The nurse coordinator monitored care.

No interactive e-care plans were available at national, state or local health service levels. A web-based GP interactive e-care plan was selected. The 


\section{Key points}

- There is a gap in the availability of interactive e-care plan solutions at the national, state and local health service levels

- Policies and leadership that encourage and enable collaboration and the safe sharing of clinical information between public cancer services and primary health care using interactive e-care plans are needed

- The feasibility, implementation and scalability of general practice interactive e-care plans for shared cancer care in follow-up needs further evaluation main concerns raised were uncertainty about the security of e-health systems not controlled by the local health service and sharing clinical information with external health providers, engaging GPs, and patient anxiety about the capacity of general practice to provide care.

The e-care plan provided a low-risk solution to sharing patient information and supported collaborative care. Challenges to share e-care plans have implications for policy and practice.

Lessons learnt: Stakeholders and the project team agreed that finding an e-health system that supported shared cancer care in follow-up and addressed the security and information sharing concerns could not all be adequately addressed at the local level. A GP interactive e-care plan provides a promising solution to a number of the barriers.

\section{Background}

In Australia, the long-term follow-up care of cancer survivors is provided by cancer services or by general practitioners (GPs), with limited communication between them. It is widely recognised that survivorship care is not optimal. ${ }^{1,2}$ The growing number of survivors (50\% to $69 \%$ over the past 33 years in Australia) ${ }^{3}$, their diverse, unmet and ongoing healthcare needs ${ }^{4}$, increasing demands on cancer services, limited resources, and a lack of integrated and coordinated care are all barriers to highquality follow-up care. ${ }^{5}$

Shared cancer care in follow-up is acceptable to patients and providers according to three Australian qualitative/descriptive studies ${ }^{6-8}$, provided there are defined roles, protocols, information sharing ${ }^{7}$, GP education $^{6,7}$, clinical leadership and patient readiness. ${ }^{6}$ A systematic review of shared survivorship care reported that it was as effective as specialist care for health and clinical outcomes and had higher patient satisfaction. ${ }^{9}$ Similar outcomes were reported in an Australian trial of follow-up colorectal cancer care provided by either specialists or GPs. ${ }^{10}$

Optimal cancer follow-up requires the prevention and surveillance for cancer spread, recurrence and new cancers; the assessment and management of the physical effects of cancer and short- and long-term impacts of treatments; the management of psychosocial issues; support for healthy lifestyle behaviours and disease prevention; management of chronic conditions; and coordination of care between specialists and primary care providers to ensure patient needs are met. ${ }^{1,11}$

Shared care in follow-up can be supported by an interactive, e-care plan that provides a collaborative space for providers and patients to communicate, share information and results, review care and track adherence. ${ }^{4,12}$ This can potentially provide optimal, person-centred, integrated and coordinated care that meets the needs of patients. ${ }^{1,11}$
To address the need to optimise shared cancer care in follow-up and close the gap in the interactive sharing of information, we established a service improvement model targeting colorectal cancer patients who had completed active treatment and agreed to shared care.

The objectives of this paper are to describe the process of developing an approach to shared cancer care in follow-up and to identify the e-health options that support an interactive e-care plan shared between a public cancer service, GPs and cancer survivors.

\section{Design and development}

Consultations were conducted with 25 stakeholders identified by the project's steering committee, based on the stakeholders' expertise in cancer or primary health care, e-health technologies and/or their ability to support the delivery of shared cancer care. Stakeholders were located in New South Wales (NSW), Australia, and were from the local health services, (including managers, specialists and nurse coordinators from cancer services [8], integrated care [3] and information and communication technologies [4]), eHealth NSW (3), a Primary Health Network (PHN) (3), GPs (2), the Translational Cancer Research Network (1) and a consumer. The objective was to design the care pathway and identify the e-health options that support an interactive care plan. Responses were collated and commonalities identified by the project team and a summary circulated.

Further consultation was conducted through a halfday workshop with 13 stakeholders (including 10 already consulted; 17 were invited), plus two researchers from the project team, to obtain consensus on the care pathway and the e-health solution to share the care plan. Participants included representatives from a Local Health District (cancer services [2], integrated care [2] and information and communication technologies [2]), the PHN (1), general practice (1), eHealth NSW (4) 
and a consumer with lived experience of cancer. The workshop provided a space for key stakeholders and the project team to collaborate, exchange ideas and obtain consensus. It followed a structured agenda and was facilitated by a researcher (JT) and a representative from eHealth NSW (JP). A summary of the issues raised and decisions was collated and distributed to stakeholders.

An ethics application was not required as this was a service improvement initiative by the South Eastern Sydney Local Health District which met the NSW Health Quality Improvement and Ethical Review policy requirements (Quality Improvement and Ethical Review GL2007_20).

\section{Outcomes of the consultation}

\section{process}

The stakeholders agreed to the cancer care pathway detailed in Figure 1. The pathway starts at the cancer service with an assessment of patient suitability and then confirmation of patient consent. The GP is invited to participate in shared care and provided with a treatment summary. The interactive e-care plan is then initiated by the GP who gives the specialist access to tailor the plan and the nurse coordinator access to monitor care and coordinate communication between the GP and specialist. Tasks are marked as completed, results and information uploaded and shared. Patients have access to add measures, notes and share documents.

Various e-health options being used by local health services to share clinical information were considered (Table 1). It was agreed at the workshop that these systems (HealtheNet, secure messaging, My Health Record) did not enable interactive collaboration and that we needed to look outside the state and federal health systems for a solution. GP e-care plans were considered. These commercial web-based systems were developed for GPs to manage care plans and are integrated with general practice clinical information systems. They had the functionality to support the sharing of an interactive care plan with cancer services.

Challenges that arose included the reluctance of local health services to share clinical information with external health providers due to a lack of clarity at the state level around sharing information using e-health tools. Security concerns about e-health systems not controlled by the local health service was also an issue.

Engaging GPs was a major issue for stakeholders involved in other integrated care projects. They perceived a lack of financial incentives for GPs under the current fee-for-service payment to be a barrier, as was introducing new technology.

It was therefore important that the interactive e-care plan worked as seamlessly as possible for GPs who would have greater interaction with the care plan, including initiating the template, providing access to team members, uploading information and communicating when tasks were completed.

Patient anxiety about the capacity of general practice to provide follow-up care and their ability to return to specialist services was raised by specialists and the consumer.

The identified challenges and solutions or workarounds and implications for policy and practice are shown in Table 2.

Figure 1. Shared care cancer plan - final pathway

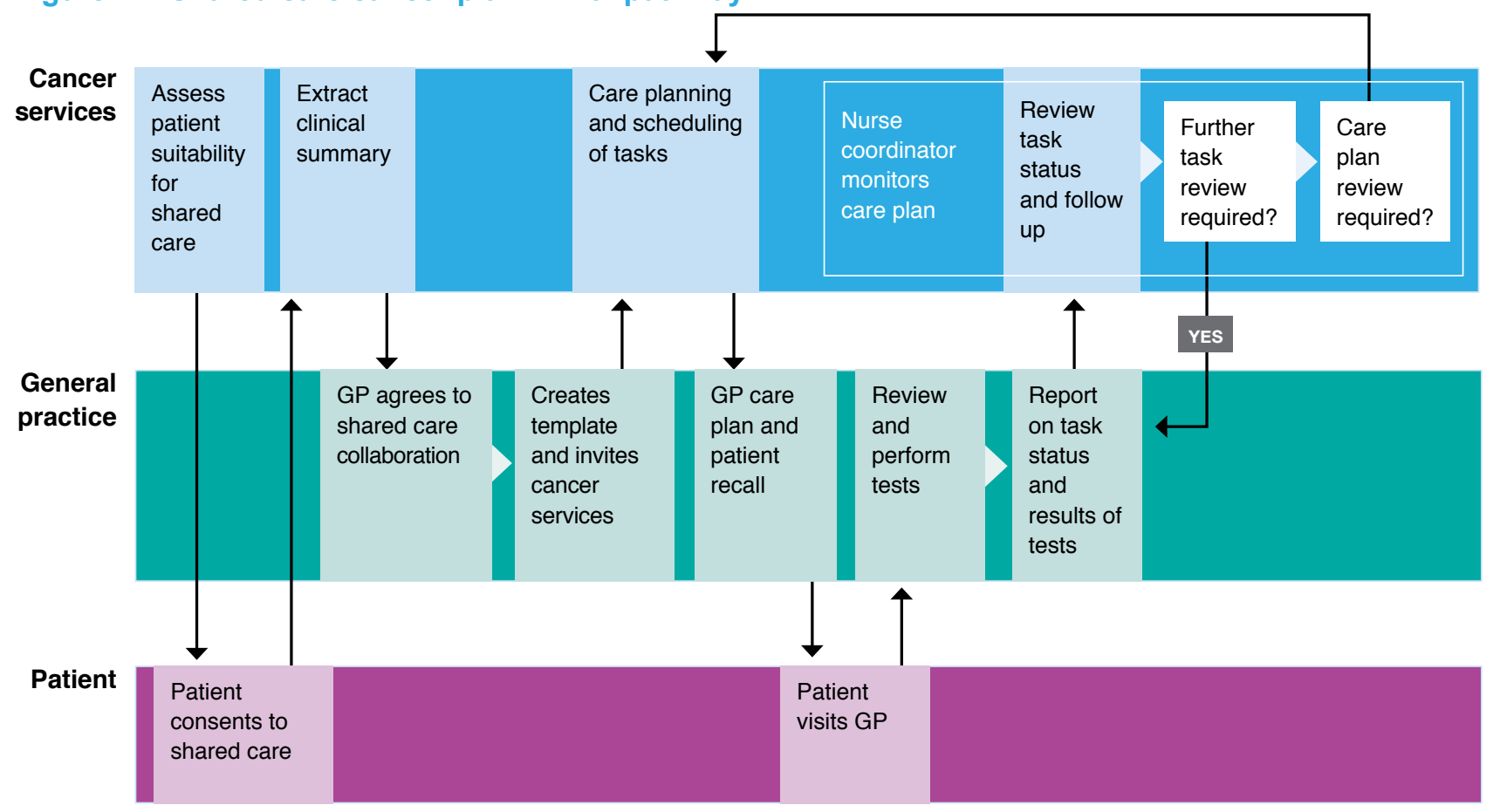


Table 1. Advantages and disadvantages of e-health options considered for sharing a follow-up care plan

\begin{tabular}{|c|c|}
\hline Options & Advantages \\
\hline $\begin{array}{l}\text { My Health Record } \\
\text { (An online summary of an } \\
\text { individual's health record) }\end{array}$ & $\begin{array}{l}\text { - } \quad \text { Patient controlled } \\
\text { - } \quad \text { Care team access } \\
\text { - } \quad \text { Can view diagnostic and pathology results } \\
\text { - } \quad \text { Integrated with general practice clinical } \\
\text { information systems }\end{array}$ \\
\hline
\end{tabular}

\section{Disadvantages}

- No care plan functionality

- Not interactive to support collaboration

- Some practices not connected to My Health Record (25\% in Central and Eastern Sydney area)

- Cancer service information systems are not integrated with My Health Record

$\begin{array}{lll}\text { Secure messaging } & \bullet & \text { Most GPs have secure messaging } \\ \text { (Argus, HealthLink and } & \bullet & \text { Integrated with general practice clinical } \\ \text { Medical Objects are } & & \text { systems }\end{array}$

the main messaging systems)

- Not interactive

- Not accessible to patients

- Version control a problem

- Cancer service would need three secure messaging systems to cater for the different systems that were not interoperable at the time

- Many allied health services do not have secure messaging

\section{HealtheNet}

(Secure sharing portal connecting a patient's medical history from information systems within the NSW Local Health Districts)
- Can upload documents from the cancer information system to HealtheNet

- Provides access to NSW Health clinicians to view My Health Record

- Care plan could be attached as the follow-up action plan to a discharge summary
- Accessible only to NSW Health employees or associates and not external care providers

- Not accessible to patients

- GPs cannot send documents through HealtheNet

- Technical development required to send the care plan through HealtheNet

- Does not support collaboration

General practice e-care plans

- Supports access and collaboration between team members and the patient

(Web-based services to manage care plans) information systems so information can be
- Integrates with general practice clinical uploaded seamlessly

- Care plan templates for different cancers can be added

- Includes prompts and notifications to coordinate and monitor care

- Some systems are compliant with NSW and Commonwealth standards

- GPs can claim the Medicare Benefits Schedule item and receive payment for generating or reviewing the care plan

- Less expensive than developing a new system

- Scalable to other cancers, conditions and services
- Not interoperable with Local Health District or general practice information systems

- Cancer staff and patients have to access and interact with the care plan through a web interface

- Off-the-shelf product does not meet all functionality requirements

\section{Discussion and conclusion}

Interactive e-care plans are not used for cancer followup care in Australia and there are no published reports of their use internationally. Care plans are paper or web-based documents, some created using care plan generators, and shared online or via email. ${ }^{15-17}$ They are not dynamic and do not facilitate team collaboration.

We identified a gap in the availability of interactive e-care plans at the national, state and local health service levels. GP interactive e-care plans may partly fill this gap. In our development process stakeholders found them to be acceptable, with the potential to support secure sharing and team collaboration. They may also be scalable for use with other types of cancer due to flexible templates and affordability.

A disadvantage was the lack of interoperability. They did not enable two-way exchange and use of information between systems and there was no integration with cancer service information systems. An absence of interoperability is a barrier to the adoption, scale-up and sustainability of health technologies. ${ }^{18}$ There was some integration with GP clinical information systems allowing clinical information to be uploaded to complete the care plan in compliance with Medicare requirements. 
Table 2. Challenges, solutions and implications for policy and practice

\begin{tabular}{l} 
Challenges \\
\hline Sourcing or developing an \\
interactive e-care plan that \\
supports collaboration and meets \\
the needs of users
\end{tabular}

the needs of users

Solution/workaround

- Using an existing lower-cost GP care planning system

Implications for policy and practice

- Consider state-led shared e-care plan systems. Need to address interoperability, security and scalability to other services and conditions

- Previous and current work on shared care planning conducted in $\mathrm{NSW}^{13}$ and planned work in Western Australia ${ }^{14}$ can inform states and territories

Addressing concerns about the security of e-health systems not controlled by state or local health services
- A security compliance check on the GP e-care plan system was conducted by the local health service

- Executive approval was obtained to pilot the GP care planning system

- Access to care plan controlled by GP with patient.

Uncertainty by health service managers as to whether clinical information can be shared using GP interactive e-care plans

Difficulty engaging GPs in integrated care
- Patient details and clinical information uploaded by GPs instead of specialists.
- Engaging GPs through their patients who opt for shared care
- State shared e-care plan systems could reduce security concerns of managers

- The need for health service-endorsed systems that comply with security and information sharing standards and policies
- Leadership to drive services and policies that support services to securely share information

- Using GP e-care plans that:

- Integrate with GP clinical information systems

- Support administration of Medicare Benefits Schedule care planning Items

- Provide GPs with:

- Access to the cancer specialist

- Rapid referral to the cancer service

- Resources and education

Patient anxiety about the capacity of general practice and access to specialist services
- Patients informed and agree to shared care

- GP education and resources provided

- Phone contact and rapid referral to cancer services available to GPs
- Flexible funding models to engage GPs in integrated and collaborative shared care

- Work towards integrated and interoperable e-care plan
- Patient anxiety a consideration in the selection of patients for shared care

- Cancer services need to support rapid access back to a specialist, and follow-up care training and resources for GPs are important
The security and information sharing concerns could not be addressed at the local service level. Patient and general practice security concerns were not raised, possibly due to the small number of consumers (1) and GPs (2) consulted. A survey with more than 1600 participants reported that although patients were willing to share clinical information they were concerned about privacy. ${ }^{19}$

A previous systematic review reported that engaging GPs was a barrier to implementing shared care. It recommended flexible, sustainable funding mechanisms and identified the need for training, roles and responsibilities for GPs. Competing GP demands and administration were other barriers connected with engagement. ${ }^{20}$
Policies and leadership that encourage and enable collaboration and the safe sharing of clinical information between public health services and primary health care using interactive e-care plans are needed. Their impact on patient outcomes and health service use also needs to be evaluated. To achieve this, a pilot study is currently underway at the St George Hospital Cancer Care Centre in Sydney to evaluate shared cancer care in follow-up and the feasibility of interactive e-care plans.

Although further improvements can be made, this cancer service improvement initiative provides service managers and state and national policy makers with a model for shared cancer care in follow-up between cancer services and general practice. 


\section{Acknowledgements}

This work is dedicated to the memory of John Lewis who contributed to the developmental work reported in this manuscript.

The project was funded by the South Eastern Sydney Local Health District The Inspiring Ideas Challenge and the Cancer Institute NSW Innovations in Cancer Control Grant.

\section{Peer review and provenance}

Externally peer reviewed, not commissioned. $\mathrm{MH}$ is an Associate Editor for Public Health Research \& Practice. He had no part in the peer-review process for this paper.

\section{Competing interests}

None declared.

\section{Author contributions}

JT was involved with managing, designing and implementing the project and was responsible for writing and reviewing the manuscript. $\mathrm{MC}$ and $\mathrm{WL}$ are cancer specialists who were involved with designing and implementing the project and writing and reviewing the manuscript. DG provided advice for the project implementation and assisted in writing and reviewing the manuscript. AD advised on digital technology in general practice, supported GP participation and provided input into the development and review of the manuscript. JP provided digital technology and health policy advice, assisted with the workshop and reviewed the manuscript. $\mathrm{MH}$ was involved in the design development and implementation of the project and writing and reviewing the manuscript.

\section{References}

1. Clinical Oncology Society of Australia. Model of survivorship care: critical components of cancer survivorship care in Australia position statement. Sydney: Clinical Oncology Society of Australia; 2016 [cited 2021 Apr 13]. Available from: www.cosa.org.au/ media/332340/cosa-model-of-survivorship-care-fullversion-final-20161107.pdf

2. National Academies of Sciences, Engineering, and Medicine; Health and Medicine Division; Board on Health Care Services; National Cancer Policy Forum. Long-term survivorship care after cancer treatment: proceedings of a workshop. Washington (DC): National Academies Press (US); 2018.
3. Australian Institute of Health and Welfare. Cancer in Australia 2019. Canberra: AlHW; 2019 [cited 2021 Apr 13]. Available from: www.aihw.gov.au/ getmedia/8c9fcf52-0055-41a0-96d9-f81b0feb98cf/ainwcan-123.pdf.aspx?inline=true

4. Emery JD, Shaw K, Williams B, Mazza D, FallonFerguson J, Varlow M, et al. The role of primary care in early detection and follow-up of cancer. Nat Rev Clin Oncol. 2014;11(1):38-48.

5. Alfano CM, Jefford M, Maher J, Birken SA, Mayer DK. Building personalized cancer follow-up care pathways in the United States: lessons learned from implementation in England, Northern Ireland, and Australia. Am Soc Clin Oncol Educ Book. 2019;39:625-39.

6. Jefford M, Kinnane N, Howell P, Nolte L, Galetakis S, Mann GB, et al. Implementing novel models of posttreatment care for cancer survivors: enablers, challenges and recommendations. Asia Pac J Clin Oncol. 2015;11(4):319-27.

7. Schütze H, Chin M, Weller D, Harris MF. Patient, general practitioner and oncologist views regarding long-term cancer shared care. Fam Pract. 2017;35(3):323-9.

8. Giles C, Nehill C, Milch V, Zorbas H. Shared follow-up care for early breast cancer - results from an Australian national demonstration project. BMC Health Serv Res. 2014;14(Suppl 2):P44.

9. Zhao Y, Brettle A, Qiu L. The effectiveness of shared care in cancer survivors-a systematic review. Int J Integr Care. 2018;18(4):2.

10. Wattchow DA, Weller DP, Esterman A, Pilotto LS, McGorm K, Hammett Z, et al. General practice vs surgical-based follow-up for patients with colon cancer: randomised controlled trial. $\mathrm{Br} \mathrm{J}$ Cancer. 2006;94(8):1116-21.

11. Nekhlyudov L, Mollica MA, Jacobsen PB, Mayer DK, Shulman LN, Geiger AM. Developing a quality of cancer survivorship care framework: implications for clinical care, research, and policy. J Natl Cancer Inst. 2019;111(11):1120-30.

12. Harris MF AJ, Crabtree BF, Levesque J-F, Miller WL, Gunn JM, et al. Interprofessional teamwork innovations for primary health care practices and practitioners: evidence from a comparison of reform in three countries. J Multidiscip Healthc. 2016;9:35-46.

13. NSW eHealth. Consolidated shared care planning solution functional and non-functional requirements, V1.0. Sydney: NSW eHealth; 2018. Available from authors.

14. Western Australia Department of Health. WA Health digital strategy 2010-2030. Perth: Government of Western Australia Department of Health; 2019 [cited 2021 Apr 13]. Available from: ww2.health.wa.gov.au/-/media/ Files/Corporate/Reports-and-publications/Digital-strategy/ Digital-Strategy-2020-2030.pdf 
15. Gramatges MM, King J, Horowitz ME, Fordis M, Poplack DG. Improving childhood cancer survivor care through web-based platforms. Oncology (Williston Park) 2018;32(1):e1-10.

16. Frick MA, Vachani CC, Bach C, Hampshire MK, ArnoldKorzeniowski K, Metz JM, et al. Survivorship and the chronic cancer patient: patterns in treatment-related effects, follow-up care, and use of survivorship care plans. Cancer. 2017;123(21):4268-76.

17. Tevaarwerk AJ, Wisinski KB, Buhr KA, Njiaju UO, Tun M, Donohue S, et al. Leveraging electronic health record systems to create and provide electronic cancer survivorship care plans: A pilot study. J Oncol Pract. 2014;10(3):e150-9.
18. Greenhalgh T, Wherton J, Papoutsi C, Lynch J, Hughes G, A'Court C, et al. Beyond adoption: a new framework for theorizing and evaluating non-adoption, abandonment, and challenges to the scale-up, spread, and sustainability of health and care technologies. J Med Internet Res. 2017;19(11):e367.

19. Abdelhamid M, Gaia J, Sanders GL. Putting the focus back on the patient: how privacy concerns affect personal health information sharing intentions. J Med Internet Res. 2017;19(9):e169.

20. Lisy K, Kent J, Dumbrell J, Kelly H, Piper A, Jefford M. Sharing cancer survivorship care between oncology and primary care providers: a qualitative study of health care professionals' experiences. J Clin Med. 2020;9(9):2991.

\section{Copyright: (c) (ㅇ) (2)}

(C) 2021 Taggart et al. This article is licensed under the Creative Commons Attribution-NonCommercial-ShareAlike 4.0 International Licence, which allows others to redistribute, adapt and share this work non-commercially provided they attribute the work and any adapted version of it is distributed under the same Creative Commons licence terms. See: www.creativecommons.org/licenses/by-nc-sa/4.0/ 